\title{
Dipole moments of poly(trimethylene oxide) chains
}

\author{
J. E. Mark and D. S. Chiu \\ Department of Chemistry and the Macromolecular Research Center, University of Michigan, Ann Arbor, \\ Michigan 48109 \\ (Received 8 March 1976) \\ Dipole moments of poly(trimethylene oxide) chains $\left[\mathrm{CH}_{2}-\mathrm{CH}_{2}-\mathrm{CH}_{2}-\mathrm{O}-\right]_{x}$ were determined as a function \\ of degree of polymerization and temperature by means of dielectric constant measurements in the \\ thermodynamically good solvent carbon tetrachloride. The mean-square dipole moment was found to be \\ directly proportional to the degree of polymerization $x$, or the number of skeletal bonds, thus \\ demonstrating the absence of excluded volume effects on the dipole moment. According to previous studies, \\ the unperturbed dimensions of this chain molecule are unusually small and essentially independent of \\ temperature; this is apparently due to the high degree of conformational randomness in the trimethylene \\ oxide repeat unit in that the two $\mathrm{C}-\mathrm{C}$ skeletal bonds prefer gauche states whereas the two $\mathrm{C}-\mathrm{O}$ bonds \\ prefer trans states. The present results indicate that this randomness has a much smaller effect on the \\ dipole moment and its temperature coefficient.
}

\section{INTRODUCTION}

Viscometric studies ${ }^{1,2}$ of poly(trimethylene oxide) $\left[\left(\mathrm{CH}_{2}\right)_{3}-\mathrm{O}-\right]_{x}$ in dilute solution indicate that this chain molecule has unusually small values of the random-coil dimensions $\left\langle\boldsymbol{r}^{2}\right\rangle_{0}$, as unperturbed by excluded volume interactions. ${ }^{3,4}$ This unusual compactness is apparently due to a high degree of conformational randomness, in that the two $\mathrm{C}-\mathrm{C}$ skeletal bonds in the four-bond repeat unit show a preference for gauche states, while the two $\mathrm{C}-\mathrm{O}$ bonds show the opposite preference for the alternative trans state. ${ }^{1,2,4-6}$ The origin of this randomness can be seen from the typical poly(trimethylene oxide) sequence<smiles>CCCCOCCCOC</smiles>

represented here in the planar all-trans conformation. Rotation to a gauche state about any skeletal bond decreases the distance of separation between a pair of atoms or groups separated by three bonds. In the case of the $\mathrm{C}-\mathrm{C}$ bonds, the species involved are a relatively small $\mathrm{O}$ atom and a $\mathrm{CH}_{2}$ group of opposite ch:ere; as might be expected, the net interaction is favorable and the energy of the gauche state is somewhat less than that of the trans. Rotation about one of the $\mathrm{C}-\mathrm{O}$ bonds, however, brings two relatively large $\mathrm{CH}_{2}$ groups into proximity and the gauche states about these bonds are significantly higher in energy than the corresponding trans state. There is thus a conformational randomness on averaging over the repeat unit as a whole. ${ }^{1,2}$ The above characteristics of the poly(trimethylene oxide) chain also have a marked effect on the temperature coefficient of its unperturbed dimensions, since increasing the temperature increases the number of trans states, of high spatial extension, about the $\mathrm{C}-\mathrm{C}$ bonds, but decreases their number about the $\mathrm{C}-\mathrm{O}$ bonds. The two effects would be expected to be partially offsetting and this is confirmed by the fact that $d \ln \left\langle r^{2}\right\rangle_{0} / d T$ of this chain molecule is found to be essentially zero. ${ }^{1}$ Finally, this high degree of conformational randomness is probably also the origin of the fact that poly(trimethylene oxide) has the lowest melting point of any member in the polyoxide series $\left[\left(\mathrm{CH}_{2}\right)_{y}-\mathrm{O}-\right]_{x} \cdot{ }^{1}$

Because of these very unusual features, it is obvious- ly of importance to investigate a number of other configuration-dependent properties of poly(trimethylene oxide). The present study focuses on the dipole moment, a statistical property known to be a very sensitive probe for the study of spatial configurations of a chain molecule. ${ }^{7-12}$ Values of the dielectric constant and index of refraction are reported for fractions of the polymer in carbon tetrachloride, a thermodynamically good solvent. Mean-square dipole moments $\left\langle\mu^{2}\right\rangle_{0}$ derived therefrom are expressed in terms of the dipole moment ratio $\left\langle\mu^{2}\right\rangle_{0} / \mathrm{nm}^{2}$, where $n$ is the number of skeletal bonds and $m^{2}$ is the average square of their dipole moments. Values of $\left\langle\mu^{2}\right\rangle_{0} / \mathrm{nm}^{2}$ determined as a function of the degree of polymerization $x$ provide an important test of the prediction that excluded volume interactions should have no effect on the dipole moments of a highly symmetric chain like poly(trimethylene oxide). ${ }^{13-15}$ In addition, results obtained as a function of temperature yield a value of the important coefficient $d \ln \left\langle\mu^{2}\right\rangle_{0} / d T^{4}$

\section{EXPERIMENTAL DETAILS}

Four fractions of poly(trimethylene oxide) having relatively narrow molecular weight distributions were employed in the present study. They were among those studied previously with regard to their unperturbed dimensions, ${ }^{1,2}$ and details of their preparation are therefore given elsewhere. ${ }^{1}$ Their values of the degree of polymerization $x$, according to number-average molecular weights as determined by osmometry in benzene at $30{ }^{\circ} \mathrm{C},{ }^{1}$ are $1120,1510,2770$, and 5040 and thus cover nearly a fivefold range in chain length.

The dielectric constant measurements were carried out using a standard DM 01 Dipolmeter operating at a fixed frequency of $2.0 \mathrm{MHz} .{ }^{15}$ The apparatus was calibrated at each temperature of interest $(20,30,40$, and $50{ }^{\circ} \mathrm{C}$ ), using chromatographic grade carbon tetrachloride, benzene, and cyclohexane. Precise values of the dielectric constant $\epsilon$ of each of these solvents are available in the literature ${ }^{16}$; the values for carbon tetrachloride, the solvent used in the poly(trimethylene oxide) solutions, are given in the second column of Table I. Five solutions, separately prepared, were used for each fraction and values of the weight fraction 
TABLE I. Values of the dielectric constant, index of refraction, and density of the solvent, carbon tetrachloride.

\begin{tabular}{llll}
\hline \hline$T,{ }^{\circ} \mathrm{C}$ & $\epsilon_{1}{ }^{2}$ & $n_{1}$ & $d_{1}, \mathrm{~g} \mathrm{~cm}^{-3}$ \\
\hline 20 & 2.236 & 1.4589 & 1.5940 \\
30 & 2.216 & 1.4544 & 1.5748 \\
40 & 2.196 & 1.4485 & 1.5565 \\
50 & 2.176 & 1.4421 & 1.5390 \\
\hline \hline
\end{tabular}

${ }^{2}$ Reference 16.

$w$ of polymer contained therein ranged from 0.007 to 0.045 . Values of $\epsilon$ determined at the cited temperatures in increasing sequence were found to be in good agreement with those obtained at the same temperatures in decreasing sequence. Refractive indices $n$ at 589 $\mathrm{m} \mu$ were measured at each temperature using a Bausch and Lomb precision refractometer of the Abbe design. The values $n_{1}$ obtained for the pure solvent are given in the third column of Table I. Values of the density $d_{1}$ of the solvent, determined by pycnometry, are given in the final column.

It was considered desirable to have an estimate of how good a solvent (in the thermodynamic sense ${ }^{3}$ ) carbon tetrachloride is for poly(trimethylene oxide). For this reason, viscosity measurements were carried out on solutions of the polymer in carbon tetrachloride at $20^{\circ} \mathrm{C}$. A Cannon-Ubbelohde viscometer was employed $^{1,2}$ and a minimum of four solutions were studied for each of the poly(trimethylene oxide) fractions.

\section{RESULTS AND DISCUSSION}

The dielectric constant data were interpreted by means of the equation of Guggenheim and $\mathrm{Smith}^{17}$

$$
\begin{aligned}
\left\langle\mu^{2}\right\rangle / n m^{2}= & \left\{27 k T M /\left[4 \pi N d_{1}\left(\epsilon_{1}+2\right)^{2} n m^{2}\right]\right\} \\
& \times\left[d\left(\epsilon-\epsilon_{1}\right) / d w-d\left(n^{2}-n_{1}^{2}\right) / d w\right],
\end{aligned}
$$

TABLE II. Summary of data and results for poly (trimethylene oxide) chains $\left[\left(\mathrm{CH}_{2}\right)_{3}-\mathrm{O}-\right]_{x}$.

\begin{tabular}{lllll}
\hline \hline,${ }^{\circ} \mathrm{C}$ & $10^{-3} x^{2}$ & $d\left(\epsilon-\epsilon_{1}\right) / d w$ & $d\left(n^{2}-n_{1}{ }^{2}\right) / d w$ & $\left\langle\mu^{2}\right\rangle / n m^{2}$ \\
\hline 20 & 1.12 & 3.410 & 0.105 & 0.423 \\
& 1.51 & 3.343 & 0.109 & 0.414 \\
& 2.77 & 3.379 & 0.113 & 0.418 \\
& 5.04 & 3.329 & 0.087 & 0.415 \\
30 & 1.12 & 3.148 & 0.127 & 0.408 \\
& 1.51 & 3.263 & 0.093 & 0.428 \\
& 2.77 & 3.139 & 0.103 & 0.410 \\
40 & 5.04 & 3.241 & 0.050 & 0.431 \\
& 1.12 & 3.100 & 0.156 & 0.424 \\
& 1.51 & 3.192 & 0.059 & 0.451 \\
& 2.77 & 3.101 & 0.135 & 0.427 \\
50 & 5.04 & 3.152 & 0.068 & 0.444 \\
& 1.12 & 2.963 & 0.128 & 0.434 \\
& 1.51 & 2.799 & 0.061 & 0.422 \\
& 2.77 & 3.008 & 0.121 & 0.442 \\
& 5.04 & 3.019 & 0.080 & 0.450 \\
\hline \hline
\end{tabular}

${ }^{2}$ Calculated from values of their number-average molecular weights; see Ref. 1, where these four fractions are designated F2, F3, F8, and F10, respectively.

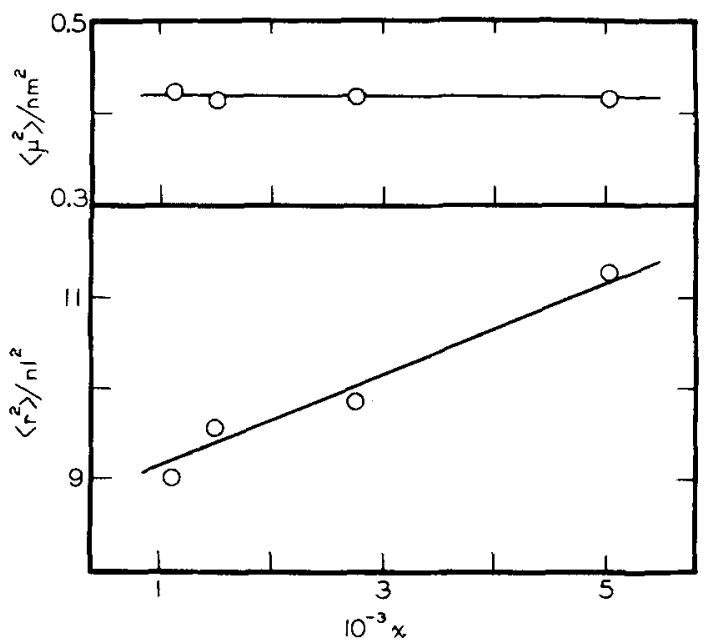

FIG. 1. The effect of the degree of polymerization on the ratios $\left\langle\mu^{2}\right\rangle / n m^{2}$ and $\left\langle r^{2}\right\rangle / n l^{2}$ determined for poly (trimethylene oxide) chains in a thermodynamically good solvent, carbon tetrachloride, at $20^{\circ} \mathrm{C}$.

where $k$ is the Boltzmann constant, $T$ is the absolute temperature, $n=4 x$ is the number of skeletal bonds, $M=x M_{0}$ (where $M_{0}=58.08$ is the molecular weight of the repeat unit), and $N$ is Avogadro's number. The average square of the bond dipoles $m^{2}=(1 / 4)\left(2 m_{\mathrm{C}-\mathrm{O}}^{2}\right)$ was calculated using the preferred value ${ }^{6} 1.07 \mathrm{D}$ for $m_{\mathrm{c}-0}$, rather than the value $0.99 \mathrm{D}$ used in an earlier study. ${ }^{5}$ Values of the derivatives $d\left(\epsilon-\epsilon_{1}\right) / d w$ and $d\left(n^{2}-n_{1}^{2}\right) / d w$ were obtained from plots of $\epsilon-\epsilon_{1}$ and $n^{2}-n_{1}^{2}$ against $w$; the results for the four poly(trimethylene oxide) fractions, at the four temperatures investigated, are given in columns 3 and 4 of Table II. The values of $\left\langle\mu^{2}\right\rangle / \mathrm{nm}^{2}$ calculated therefrom are given in the final column of Table II. The results at any of the temperatures indicate that $\left\langle\mu^{2}\right\rangle / \mathrm{nm}^{2}$ is independent of the degree of polymerization $x$; this is illustrated graphically in the upper portion of Fig. 1, which pertains to $20^{\circ} \mathrm{C}$.

The importance of this independence can be established by detailed consideration of the viscosity data obtained in the present study. Intrinsic viscosities $[\eta]$ for poly(trimethylene oxide) in carbon tetrachloride at $20^{\circ} \mathrm{C}$ were calculated from these data in the usual man$\mathrm{ner}^{3}$; the values for the four fractions were found to be $0.822,1.036,1.592$, and 2.484 d liter $\mathrm{g}^{-1}$. The values of $\lfloor\eta]_{\Theta}$ for the same fractions in a $\Theta$ solvent (cyclohexane at $\left.27.0^{\circ} \mathrm{C}\right),{ }^{2}$ in which excluded volume effects are known to be nullified, ${ }^{3}$ are $0.275,0.316,0.465$, and 0.591 diter $\mathrm{g}^{-1}$. The extent to which the chain dimension $\left\langle r^{2}\right\rangle$ is affected by excluded volume interactions is given by the chain expansion factor ${ }^{3} \alpha=\left[\left\langle r^{2}\right\rangle /\right.$ $\left.\left\langle r^{2}\right\rangle_{0}\right]^{1 / 2}$, where, as mentioned earlier, $\left\langle r^{2}\right\rangle_{0}$ is the corresponding dimension as unperturbed by such interactions. According to theory $\mathrm{y}^{3,18}$

$$
\left.\alpha=\left[[\eta] /[\eta]_{\Theta}\right)\left(\Phi_{\Theta} / \Phi\right)\right]^{1 / 3},
$$

where the ratio of hydrodynamic constants has the value $(2.5 / 2.1) .^{18,19}$ Values of the ratio $\left\langle r^{2}\right\rangle / n l^{2}$, where $l^{2}$ is the average square of the skeletal bond length, may therefore be obtained from the calculated values of $\alpha$ and the previously reported value 3.86 for the characteristic ratio $\left\langle r^{2}\right\rangle_{0} / n 1^{2}$. These results are shown in 
the lower portion of Fig. 1 and clearly demonstrate the presence of large excluded volume interactions in that $\left\langle r^{2}\right\rangle / n l^{2}$ is much larger than $\left\langle r^{2}\right\rangle_{0} / n l^{2}$ and increases markedly with $x$ over the range covered in the present experiments. Thus, carbon tetrachloride is a "thermodynamically good" solvent ${ }^{3}$ for poly(trimethylene oxide) in the vicinity of room temperature. The fact that $\left\langle\mu^{2}\right\rangle / n m^{2}$ is independent of $x$ under the same conditions therefore demonstrates that excluded volume interactions have no effect on the dipole moments of poly(trimethylene oxide), as has been predicted for chain molecules having repeat units of such high symmetry. $13-15$

Because of the absence of excluded volume effects on the dipole moment of poly(trimethylene oxide), $\left\langle\mu^{2}\right\rangle /$ $\mathrm{nm}^{2}$ could equally well be written as the unperturbed ratio $\left\langle\mu^{2}\right\rangle_{0} / \mathrm{nm}^{2}$. Therefore, these experimental values of the dipole moment ratio may be compared directly to values ${ }^{5,6}$ calculated using the rotational isomeric state theory of chain configurations. ${ }^{4}$ For a temperature in the vicinity of $20^{\circ} \mathrm{C},\left\langle\mu^{2}\right\rangle_{0} / n m^{2}$ was calculated to be $\sim 0.40$ in a preliminary study ${ }^{5}$ and 0.38 in some more reliable calculations ${ }^{6}$ which have recently been carried out. The experimental value 0.42 obtained for this ratio in the present study is thus in good agreement with theory.

In order to estimate a value of $d \ln \left\langle\mu^{2}\right\rangle_{0} / d T$ from the present experimental data, the dipole moment ratio was averaged over $x$ at each temperature. The logarithm of $\left\langle\mu^{2}\right\rangle_{0} / \mathrm{nm}^{2}$ thus averaged is shown as a function of temperature in Fig. 2. The line shown was located by least-squares analysis and yields the value $10^{3} d \ln \left\langle\mu^{2}\right\rangle_{0} /$ $d T=1.8$, with a relatively large uncertainty of \pm 0.4 $\mathrm{K}^{-1}$. This experimental result is also in satisfactory agreement with the results of the two theoretical studies already cited, which yield $1.4^{5}$ and $2.1^{B}$ for this coefficient.

As mentioned above, poly(trimethylene oxide) is a chain molecule having a great deal of conformational randomness. The effect of this randomness on configuration-dependent properties is illustrated by the results given in Table III for poly(trimethylene oxide) $\left(\mathrm{POM}_{3}\right)$ and its immediate neighbors in the $\left[\left(\mathrm{CH}_{2}\right)_{y}-\mathrm{O}-\right]_{x}$ polyoxide series, namely polyoxyethylene (POE) , $\left[\left(\mathrm{CH}_{2}\right)_{2}-\mathrm{O}-\right]_{x}$ and poly (tetramethylene oxide) $\left[\left(\mathrm{CH}_{2}\right)_{4}-\right.$ $\mathrm{O}-]_{x}$. The first two rows of the table document the fact

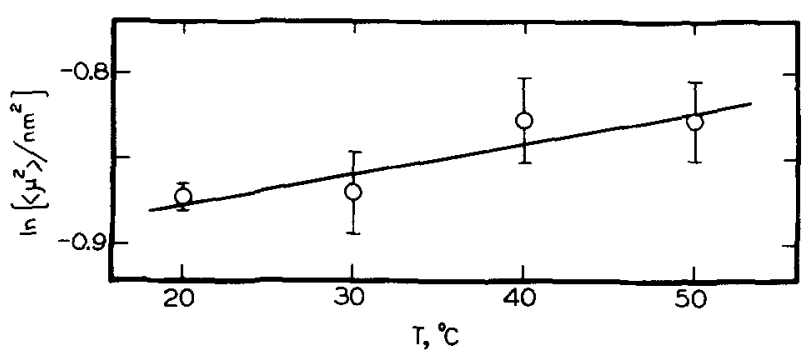

FIG. 2. The temperature dependence of the dipole moment ratio of poly (trimethylene oxide) in carbon tetrachloride. Each point represents the average value of $\left\langle\mu^{2}\right\rangle / \mathrm{nm}^{2}$ obtained for the four fractions studied, and the accompanying vertical bar specifies the average deviation from this value.
TABLE III. Some configuration-dependent properties of poly (trimethylene oxide) and its immediate neighbors, polyoxyethylene and poly (tetramethylene oxide), in the polyoxide series $\left[\left(\mathrm{CH}_{2}\right)_{y}-\mathrm{O}-\right]_{x}$.

\begin{tabular}{lllc}
\hline & $\begin{array}{l}\mathrm{POE}^{2} \\
(y=2)\end{array}$ & $\begin{array}{c}\mathrm{POM}_{3} \\
(y=3)\end{array}$ & $\begin{array}{c}\mathrm{POM}_{4}^{2} \\
(y=4)\end{array}$ \\
\hline$\left\langle r^{2}\right\rangle_{0} / n l^{2}$ & 5.2 & $3.9^{\mathrm{b}}$ & 6.1 \\
$10^{3} d \ln \left\langle r^{2}\right\rangle_{0} / d T$ & 0.23 & $0.08^{\mathrm{c}}$ & -1.3 \\
$\left\langle\mu^{2}\right\rangle_{0} / n l^{2}$ & 0.51 & $0.42^{\mathrm{d}}$ & 0.50 \\
$10^{3} d \ln \left\langle\mu^{2}\right\rangle_{0} / d T$ & 2.6 & $1.8^{\mathrm{d}}$ & 2.7 \\
\hline
\end{tabular}

${ }^{2}$ Experimental results on polyoxyethylene and poly (tetramethylene oxide) are summarized in Ref. 6 .

${ }^{b}$ Reference 2 .

'Reference 1.

dPresent study.

that poly(trimethylene oxide) has a much smaller value of the characteristic ratio than the other two polymers, and has unperturbed dimensions which are essentially independent of temperature. These characteristics are apparently due to the fact that all four skeletal bonds of the repeat unit contribute in the averaging process giving $\left\langle r^{2}\right\rangle_{0}{ }^{4}$ Thus, the conformational randomness as sociated with the fact that two of these skeletal bonds prefer gauche states whereas the other two prefer trans states does have a marked effect on $\left\langle r^{2}\right\rangle_{0}$. In the case of the dipole moment ratio, however, the only contributions to $\left\langle\mu^{2}\right\rangle_{0}$ come from the two polar C-O bonds of the repeat unit, both of which show a strong preference for trans states. The conformational randomness characteristic of the repeat unit as a whole therefore has a much smaller effect on $\left\langle\mu^{2}\right\rangle_{0} / \mathrm{nm}^{2}$, as is shown in the third row of Table III.

This difference shows up more strikingly in the temperature dependence of $\left\langle r^{2}\right\rangle_{0}$ and $\left\langle\mu^{2}\right\rangle_{0}$. The coefficient $d \ln \left\langle r^{2}\right\rangle_{0} / d T$ for poly(trimethylene oxide) is unusually small, presumably because an increase in temperature causes an increase in the number of spatially extended trans states about $\mathrm{C}-\mathrm{C}$ bonds, but decreases their number about the $\mathrm{C}-\mathrm{O}$ bonds, and the two effects at least partially offset one another. ${ }^{1}$ There is much less attenuation between the effects of these two conformational transitions on $\left\langle\mu^{2}\right\rangle_{0}$ since the skeletal bonds experiencing an increase in the number of trans states upon an increase in temperature are always the nonpolar $\mathrm{C}-\mathrm{C}$ bonds, and those experiencing a decrease in these states are always the polar $\mathrm{C}-\mathrm{O}$ bonds. Thus, the coefficient $d \ln \left\langle\mu^{2}\right\rangle_{0} / d T$ for poly(trimethylene oxide) would not be expected to be unusually small. This is confirmed by the experimental results presented in the last row of Table III, where $d \ln \left\langle\mu^{2}\right\rangle_{0} / d T$ for poly(trimethylene oxide) is seen to be large and positive, as are the values reported for its immediate neighbors in the polyoxide series, polyoxyethylene, and poly(tetramethylene oxide).

\section{ACKNOWLEDGMENTS}

It is a pleasure to acknowledge financial support provided by the National Science Foundation, and a post- 
doctoral fellowship awarded D.S.C. by the Macromolecular Research Center of the University of Michigan.

${ }^{1}$ Y. Takahashi and J. E. Mark, "Randon-Coil Dimensions of Poly(trimethylene oxide), an Unusually Compact Molecule," J. Am. Chem. Soc. (to be published).

${ }^{2}$ D. S. Chiu, Y. Takahashi and J. E. Mark, "Dimensions of Poly(trimethylene oxide) Chains in a theta Solvent," Polymer (to be published).

${ }^{3} \mathrm{P}$. J. Flory, Principles of Polymer Chemistry (Cornell University, Ithaca, 1953).

${ }^{4}$ P. J. Flory, Statistical Mechanics of Chain Molecules (Interscience, New York, 1969).

${ }^{5}$ J. E. Mark, J. Polym. Sci. B 4, 825 (1966).

${ }^{6}$ A. Abe and J. E. Mark, "Conformational Energies, and the Random-Coil Dimensions and Dipole Moments of the Polyoxides $\left.\mathrm{CH}_{3} \mathrm{O}\left(\mathrm{CH}_{2}\right)_{y} \mathrm{O}\right]_{x} \mathrm{CH}_{3}$," J. Am. Chem. Soc. (to be published).

${ }^{7} J$. E. Mark, J. Chem. Phys. 56, 451, 458 (1972).

${ }^{8}$ J. E. Mark, J. Am. Chem. Soc. 94, 6645 (1972).
${ }^{9}$ J. E. Mark, Polymer 14, 553 (1973).

${ }^{10}$ J. E. Mark, Acc. Chem. Res. 7, 218 (1974).

${ }^{11}$ J. H. Ko and J. E. Mark, Macromolecules 8, 869, 874 (1975).

${ }^{12}$ J. E. Mark, "Configuration-Dependent Properties of Chain Molecules," J. Polym. Sci. (to be published).

${ }^{13}$ K. Nagai and T. Ishikawa, Polym. J. 2, 416 (1971).

${ }^{14}$ M. Doi, Polym. J. 3, 252 (1972).

${ }^{15}$ S. C. Liao and J. E. Mark, J. Chem. Phys. 59, 3825 (1973).

${ }^{16} \mathrm{R}$. Mecke and K. Rosswog, Z. Electrochem. 60, 47 (1956); J. Timmermans, Physico-Chemical Constants of Pure Organic Compounds (Elsevier, Amsterdam, 1965), Vols, 1 and 2; H. H. Landolt, Landolt-Börnstein: Zahlenwerte und Funktionen aus Phys., Chem., Astrom., Geophys, und Tech. (Springer, Berlin, 1959), Vol. II, Part 6.

${ }^{17}$ E. A. Guggenheim, Trans. Faraday Soc. 45, 714 (1949); 47, 573 (1951); J. W. Smith, ibid. 46, 394 (1950).

${ }^{18}$ S. Bluestone, J. E. Mark, and P. J. Flory, Macromolecules 7. 325 (1974).

${ }^{19}$ D. McIntyre, A. Wims, L. C. Williams, and L. Mandelkern, J. Phys. Chem. 66, 1932 (1962); G. C. Berry, J. Chem. Phys. 46, 1338 (1967). 